\title{
MEASUREMENT OF LONGITUDINAL INSTABILITY THRESHOLD IN THE PF-AR
}

\author{
Takao Ieiri and Takashi Obina \\ KEK, 1-1 Oho, Tsukuba-shi, Ibaraki, 305-0801 Japan
}

Abstract

A single bunch longitudinal instability was experimentally investigated in the PF-AR under the condition that the number of cavity-cell which greatly contributed to longitudinal impedance was changed. The bunch length was measured as a function of the bunch current. Hysteresis phenomena in bunch lengthening process were discovered in the former condition of fully installed cavities. The present condition of reduced cavity-cells to $3 / 4$ indicated an abrupt increase of the bunch length together with the energy spread at a bunch current. The instability threshold current was measured as a function of the bunch length. Measured results are consistent with Oide's criterion.

\section{INTRODUCTION}

A single bunch longitudinal instability is discussed with potential-well distortion [1] and microwave or turbulent instability [2] in electron storage rings. The Ha $\ddot{i}$ ssinski equation gives an exact solution to the bunchshape deformation if the wake potential is determined. As the beam current increases, turbulent instability may be excited above a threshold. Turbulent instability causes bunch lengthening together with energy spreading, which has been almost explained by the Boussard criterion [3]. However, the Boussard criterion is only useful for long bunch length or an inductive wake. Oide and Yokoya [4] and D'yachkov and Baartman [5] independently investigated the longitudinal instability using simple wakefields models. It is important to study an instability for actual wakefields in storage rings.

Former experiments [6,7] showed that hysteretic behavior was observed in bunch lengthening process, where the PF-AR had fully installed 88 cavity-cells. The hysteresis disappeared when the number of the cavity-cell reduced to 44 without changing other components. It was pointed out that the hysteresis was caused by a composit wake produced by the cavity and bellows. Now the PF-AR has 66 cells and it is interesting to investigate how the bunch length behaves. This note experimentally evaluates the threshold of a longitudinal instability when the impedance was changed.

\section{PF - A R}

The PF-AR is a storage ring of $377 \mathrm{~m}$ in circumference. There are four long straight sections, each with a length of approximately $20 \mathrm{~m}$. Two straight lines, east and west parts, are prepared for rf cavities.
Alternating periodic structure (APS) cavities with multicells [8] were installed to raise the beam energy from 2.5 Gev to $6.5 \mathrm{GeV}$. Each APS cavity has 11 accelerating cells. The PF-AR usually runs with a single bunch operation. The main parameters at the energy of 2.5 $\mathrm{GeV}$ are listed in Table I.

The impedance of the PF-AR is dominated by the APS cavities and non-shielded bellows. The wake potential of the cavity showed a resistive type for bunch length of 1.5 $\mathrm{cm}$. On the other hand, the wake of 130 bellows with 12 convolutions was inductive. Thus a bunch is affected by the two different types of wakes. The number of the cavities was historically changed according to required beam energy. When the AR was used as an injector for the TRISTAN main ring, the number of the cavity-cells was 88 . Now the number is 66 .

Table I : Main parameters of the PF-AR.

\begin{tabular}{lll}
\hline $\mathrm{E}$ & Beam Energy & $2.5 \mathrm{GeV}$ \\
$\mathrm{f}_{\mathrm{rf}}$ & Accelerating Frequency & $508.58 \mathrm{MHz}$ \\
$\mathrm{h}$ & Harmonic Number & 640 \\
$\mathrm{~V}_{\mathrm{c}}$ & Accelerating Cavity Voltage & $0.5-4.0 \mathrm{MV}$ \\
$\mathrm{f}_{\mathrm{S}}$ & Synchrotron Frequency & $10-36 \mathrm{kHz}$ \\
$\alpha_{p}$ & Momentum Compaction & 0.0129 \\
$\sigma_{\varepsilon 0}$ & Natural Energy Spread & $4.4 \times 10^{-4}$ \\
$\tau_{d}$ & Longitudinal Damping Time & $21.6 \mathrm{~ms}$ \\
$\sigma_{z 0}$ & Natural rms Bunch Length & $0.8-3.0 \mathrm{~cm}$ \\
\hline
\end{tabular}

\section{MEASUREMENT}

The bunch length was measured both from the beam spectrum and by a streak camera. A streak camera took a bunch profile and measured its FWHM(full width half maximum). An rms bunch length was obtained in real time from the spectrum. The bunch length was measured as a function of the bunch current at constant cavity voltage, $\mathrm{V}_{\mathrm{c}}=1.5 \mathrm{MV}$. Figure 1 shows rms bunch length as a function of the bunch current. The bunch length gradually increases as the bunch current increases, where the natural bunch length is $1.3 \mathrm{~cm}$. When the bunch current reaches $8.0 \mathrm{~mA}$, the bunch length abruptly increases from $2.0 \mathrm{~cm}$ to $2.8 \mathrm{~cm}$. Next, the bunch length was measured as a function of cavity voltage starting at bunch current of $8.7 \mathrm{~mA}$. The bunch length was $2.9 \mathrm{~cm}$ at $\mathrm{V}_{\mathrm{c}}=1.5 \mathrm{MV}$ and suddenly decreased to $1.9 \mathrm{~cm}$ when the 
$\mathrm{V}_{\mathrm{c}}$ reached 1.7 MV. The bunch length has two values around $\mathrm{V}_{\mathrm{c}}=1.65 \mathrm{MV}$ as shown in Fig. 2. The bunch length went and came back between two separate values there. Though a hysteresis observed in the previous condition [6,7] disappeared, the flip-flop phenomenon was observed instead.

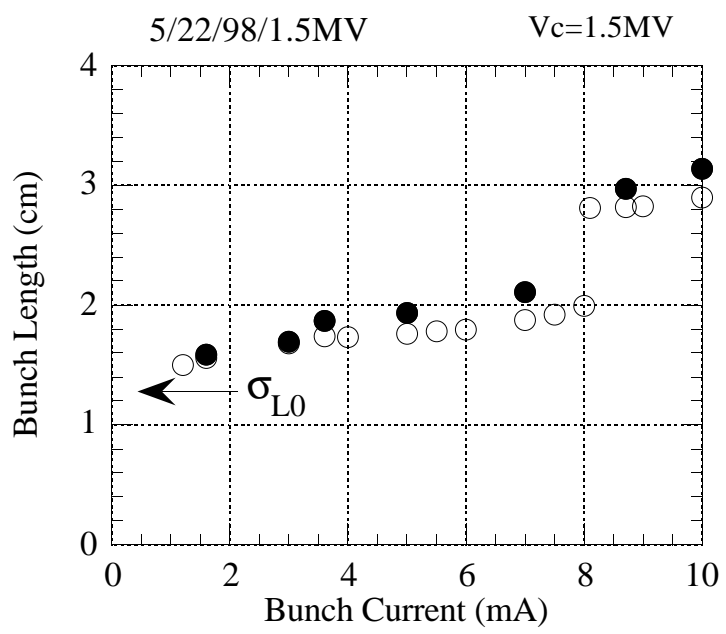

Figure 1: Bunch length vs bunch current. Dots are by streak and circles are from the spectrum.

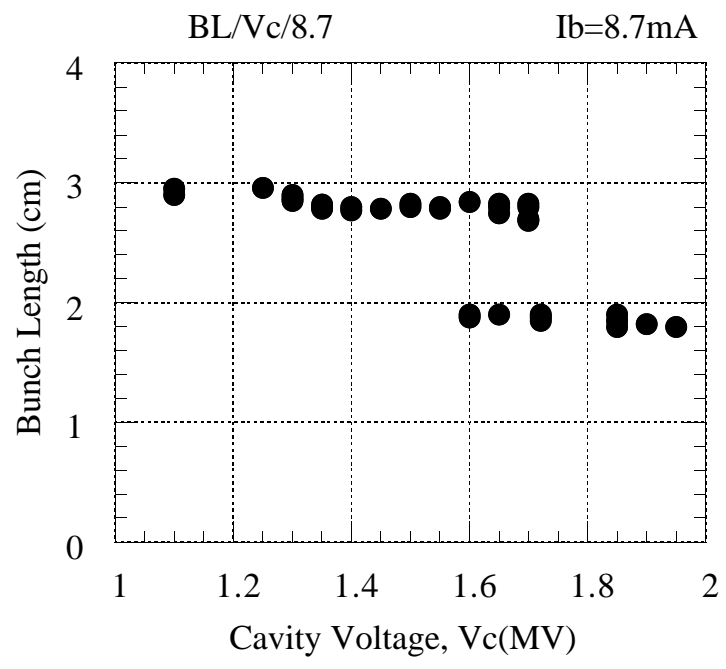

Figure 2: Bunch length as a function of cavity voltage.

A change of the beam energy spread was estimated from the quantum lifetime. The quantum lifetime is given by the ratio between the energy acceptance formed by the cavity voltage and the energy spread. However, the cavity voltage is affected by other effects. The net accelerating voltage $V_{a}$ should compensate the energy loss; the radiation energy loss of $V_{\text {rad. }}=0.15 \mathrm{MeV}$ and higher-order-mode (HOM) loss. The energy loss per turn is given by

$$
V_{a}=V_{c} \cos \phi=V_{\text {rad. }}+k T_{r} I_{b},
$$

where $\phi$ is the synchronous phase angle, $k$ is the loss parameter depending on the bunch length and $T_{r}$ is the revolution time. The loss parameter of the PF-AR is esitimated to be $25 \mathrm{~V} / \mathrm{pC}$ at the bunch length of $2.0 \mathrm{~cm}$. The loss parameter is mainly caused by the APS cavity. Moreover, the cavity voltage should ensure a phase stability condition due to beam loading in a cavity. A stored maximum current is expressed by

$$
I_{b m}=\frac{2 V_{c} \sin \phi}{\sin 2(\phi-\alpha)} \frac{1}{\left(\frac{R}{Q}\right) Q_{L}} .
$$

Here, $\alpha$ is the tuning offset angle, $R$ the shunt resistance and $Q_{L}$ the loaded $\mathrm{Q}$ value of a cavity. From eq.(1) and eq.(2), the cavity voltage is increased to be proportional to square root of the beam current under constant energy loss. A calculated voltage is shown in Fig. 3 including the HOM loss. The HOM loss was estimated from the ABCI code [9] based on measured bunch length data. A playeau in the voltage seen above $8 \mathrm{~mA}$ in Fig. 3 is due to an abrupt increase of the bunch length. A measurement was carried out by slowly reducting the cavity voltage while keeping a bunch in a bucket. The beam lifetime was above 60 minutes if an instability did not take place. As the cavity voltage gradually lowered, the lifetime slowly decreased. When the voltage reached $V_{c}=0.72 \mathrm{MV}$ at bunch current of 5.0 $\mathrm{mA}$, the lifetime suddenly reduced to less than 1 minute and a beam was lost. The minimum cavity voltage with which a beam was lost was measured as a function of the bunch current as shown in Fig. 3. The measured voltage approximately agrees with the calculated one below 7 mA. However, the measurement requires $30 \%$ higher voltage than the calculated value. This extra voltage should be caused by an increase of the energy spread. The increase of the energy spread corresponds to an abrupt increase of the bunch length.

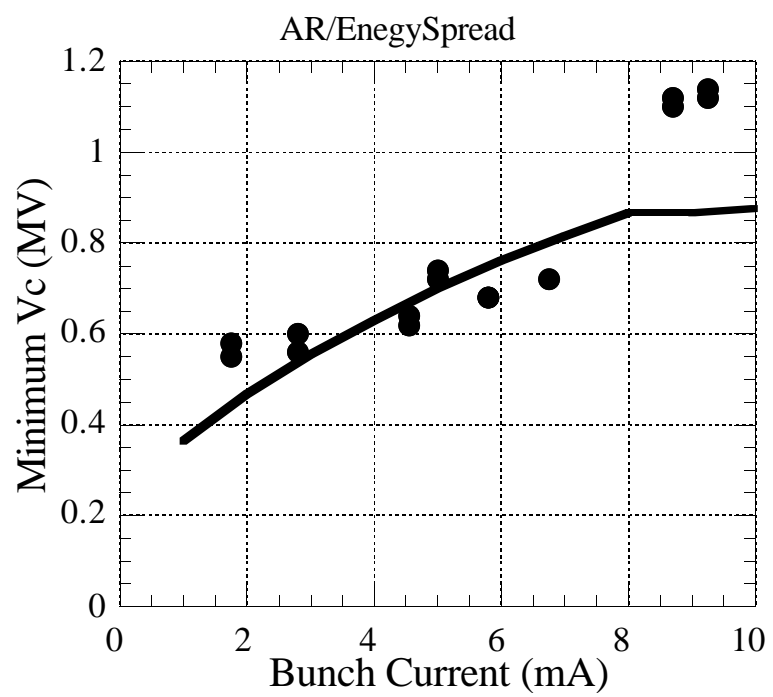

Figure 3: Measured minimum cavity voltage determined from the beam lifetime as a function of the bunch current. The solid line shows estimated voltage including HOM effects. 


\section{DISCUSSION}

A threshold current for the longitudinal instability is clearly characterized by an abrupt increase of the bunch length and of the energy spread. The threshold current is plotted as a function of the bunch length as shown in Fig. 4 together with calculated values obtained using a code [4]. The measured current decreases from $8.5 \mathrm{~mA}$ to 7.1 $\mathrm{mA}$ though the bunch length increases from $1.8 \mathrm{~cm}$ to $2.5 \mathrm{~cm}$. The calculated threshold current takes a minimum around the bunch length of $2.0 \mathrm{~cm}$. Though they have a disparity of about $30 \%$, they are consistent with each other.

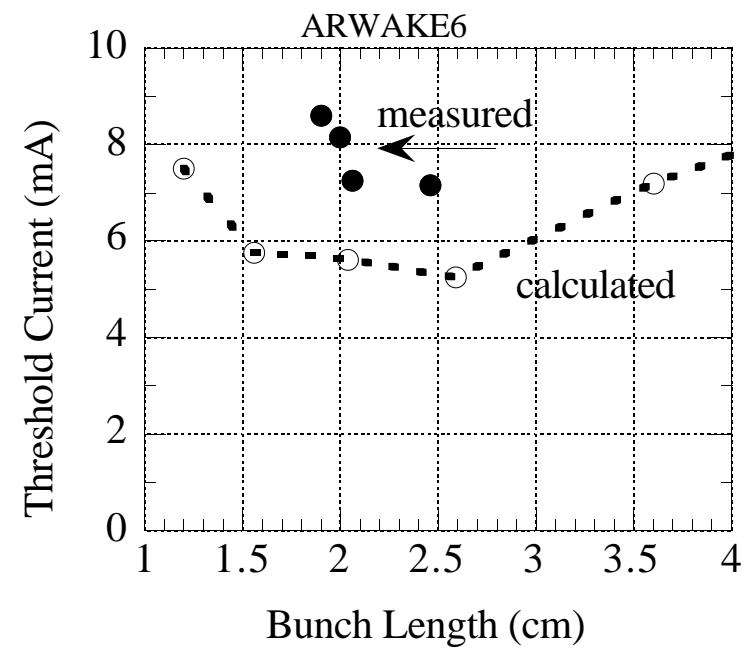

Figure 4: Threshold current as a function of the bunch length. Dots are measured and circles calculated. The bunch length is a value just before an instability.

On the other hand, Oide et al. indicated that the threshold current has a minimum in a region that the normalized resonant frequency $\omega_{0} \sigma_{z} / c$ is around 0.7, where $\omega_{0}$ is the resonant frequency of a model impedance [4]. The region is dominated by a resistive wake. The impedance of the PF-AR also shows a resistive wake produced by the multi-cell cavity. Figure 5 indicates that the threshold curent as a function of the number of cavitycell under the same synchrotron tune. The number of cavity-cell represents an intensity of a resistive wake. The experimental result suggests that the threshold decreases linearly as the resistive wake increases. From a theoretical aspect, Oide derived a simple stability condition [10] which represents

$$
k L \geq \frac{2}{9} k R q_{0} .
$$

Here, $\mathrm{k}$ is a beam intensity, $\mathrm{L}$ represents a pure-inductive wake, $\mathrm{R}$ represents a pure-resistive wake and $q_{0}$ an equilibrium position of a bunch. The stability condition suggests that an inductive wake raises threshold current but a resistive part strenghtens an instability. The experimental result is consistent with the stability condition. In conclusion, the longitudinal instability threshold current of the PF-AR characterized by a resistive wake behaves in different variations than those observed in many other storage rings, which has been already predicted by Oide et al. except the hysteresis and the flipflop phenomena.

The authors thank Prof. Z.Y.Guo of IHEP for promoting the energy spread measurement and Prof. K.Oide for encouragements of this work.

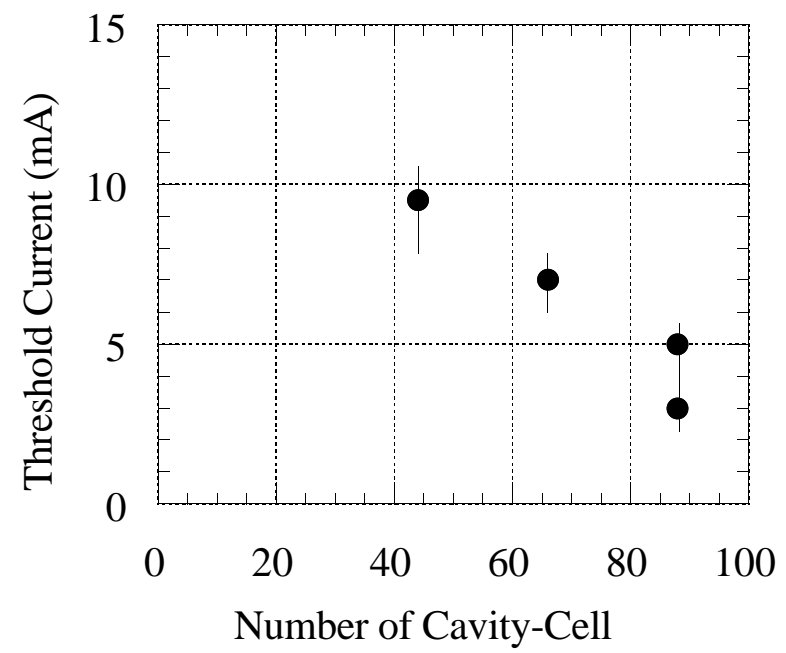

Figure 5: Threshold current as a function of the number of cavity-cell under the same synchrotron tune of 0.021 .

\section{REFERENCES}

[1] J. Ha $\ddot{i}$ ssinski, Nuovo Cimento 18B No.1, (1973)72.

[2] J.M.Wang and C.Pellegrini, Proc. of the 11th Int. Conf. on High-Energy Accelerator, CERN (1980)554.

[3] D. Boussard, LAB II / RF / Int. /75-2 (1975).

[4] K. Oide and K. Yokoya, KEK Prepr. 90-10 (1990).

[5] M. D'yachkov and R. Baartman, Proc. 4th European Particle Accelerator Conf., London, (1994)1075.

[6] T. Ieiri, Nucl. Instrum. Methods A 329 (1993)371.

[7] T. Ieiri, Jpn. J. Appl. Phys. 36 (1997)6965.

[8] T. Higo et al., Proc. 5th Symp. Accelerator Science and Technology, Tsukuba, (1984)114.

[9] Y. H. Chin, LBL-35258 UC-414 (1994).

[10] K. Oide, Particle Accelarator 51 (1995) 43. 